\title{
Desempenho de vacas Jersey suplementadas com diferentes fontes lipídicas na fase inicial da lactação
}

\author{
José Laerte Nörnberg${ }^{1}$, Jorge López ${ }^{2}$, Waldyr Stumpf Júnior ${ }^{3}$, Patricia Barcellos Costa ${ }^{4}$, \\ Jorge Schafhäuser Júnior ${ }^{5}$
}

\footnotetext{
${ }^{1}$ Departamento de Tecnologia e Ciência de Alimentos (DTCA) - Universidade Federal de Santa Maria (UFSM), 97105-900, Santa Maria, RS ${ }^{2}$ Departamento de Zootecnia da Universidade Federal do Rio Grande do Sul (UFRGS), Porto Alegre-RS. Pesquisador do CNPq.

${ }^{3}$ Centro de Pesquisa Agropecuária de Clima Temperado (CPACT) - EMBRAPA, Pelotas-RS.

${ }^{4}$ Doutora em Zootecnia - Universidade Federal de Viçosa (UFV), Viçosa-MG.

${ }^{5}$ Universidade da Região da Campanha - URCAMP, Alegrete - RS.
}

RESUMO - Este trabalho foi realizado com o objetivo de avaliar o efeito da inclusão de três fontes lipídicas (6\% de EE total) em dietas com teores semelhantes de proteína, fibra e lipídios sobre o consumo voluntário, a produção de leite e a eficiência alimentar de vacas Jersey na fase inicial da lactação. Foram utilizadas oito vacas multíparas em estágio próximo ao pico de lactação, com $420 \mathrm{~kg}$ de PV e produção média de $20 \mathrm{~kg}$ de leite (corrigida para 3,5\% de gordura), estabuladas em baias individuais. Os tratamentos foram avaliados em dois quadrados latinos (4 x 4) com períodos de 28 dias, conforme descritos a seguir: CON - dieta controle, sem inclusão de lipídios; GPC - dieta com gordura protegida comercial (sais de cálcio de ácidos graxos de óleo de palma); FAIO - dieta com farelo de arroz integral e óleo de arroz; FAIS - dieta com farelo de arroz integral e sebo bovino. Utilizaram-se concentrados à base de grãos de milho moídos e farelo de soja e, como volumosos, silagem de milho e feno de alfafa (1:1, na MS) Os concentrados foram fornecidos três vezes ao dia, separadamente dos volumosos. Os volumosos foram fornecidos à vontade, procurando-se manter a proporção de $55 \%$ em relação ao concentrado. A interpretação estatística foi feita por meio de análise de variância dos valores médios de cada tratamento aplicando-se o teste F. Os resultados comprovaram que as fontes lipídicas estudadas não afetam o consumo voluntário de MS e proporcionam maior produção de leite, de modo que a fonte de sais cálcicos de ácidos graxos de óleo de palma promoveu, também, maior produção de leite corrigida para 3,5\% de gordura e melhorou a eficiência alimentar.

Palavras-chave: farelo de arroz, leite, óleo, sais cálcicos de ácidos graxos, sebo

\section{Performance of early lactating Jersey cows supplemented with different lipid sources}

\begin{abstract}
The objective of this trial was to evaluate the effect of supplementing diets with similar protein, fiber and lipid contents with different lipid sources on dry matter intake, milk yield and feed efficiency of early lactating Jersey cows. Eight multiparous cows averaging $420 \mathrm{~kg}$ of body weight and daily yield of $20 \mathrm{~kg}$ of $3.5 \%$ fat corrected milk (FCM) were randomly assigned to two replicated 4 x 4 Latin squares. Each experimental period lasted four weeks and means were compared by F-test. Cows were fed the following diets: CON- control diet without lipid supplementation; RPF- basal diet supplemented with a commercial rumen-protected fat source (calcium salts of fatty acids from palm oil); RBRO- basal diet supplemented with rice bran and rice oil; RBT- basal diet supplemented with rice bran and tallow. The concentrate portion of the diet contained ground corn and soybean meal while the forage portion was composed by corn silage and alfalfa hay (1:1, dry matter basis) Concentrate was fed three times a day and forage ad libitum. Diets were formulated to contain a forage:concentrate ratio of 55:45. Supplementation with different dietary lipid sources did not affect dry matter intake but increased milk yield compared to the CON diet. In adittion, both FCM and feed efficiency were greatest on cows fed the RPF diet.
\end{abstract}

Key Words: calcium salts of fatty acids, milk, oil, rice bran, tallow

\section{Introdução}

O consumo de energia é a principal limitação para a produção de leite, sendo determinado pela concentração energética da dieta e por sua taxa de consumo. Com o objetivo de atender às necessidades energéticas de vacas em lactação, o uso de lipídios na alimentação desses animais têm sido recomendado para aumentar a densidade energética das dietas e evitar os efeitos prejudiciais de altas quantidades de concentrados ricos em amido sobre o ambiente ruminal (Doreau \& Chilliard, 1997). As gorduras também têm potencial para melhorar a eficiência de utilização 
da energia, uma vez que os ácidos graxos pré-formados de origem dietética são incorporados diretamente na gordura do leite, sem a perda de calor associada à síntese de ácidos graxos, poupando energia para outras funções produtivas da glândula mamária e melhorando a eficiência na produção de ATP a partir de ácidos graxos de cadeia longa em comparação ao acetato, fonte energética fundamental nos ruminantes (Doreau et al., 1991; Chilliard, 1993; Garnsworthy, 1997).

Entretanto, a resposta produtiva de vacas leiteiras à suplementação com fontes lipídicas é bastante variável, de $-4,4$ a 9,6 kg/dia de leite $/ \mathrm{kg}$ de lipídio adicionado à dieta (Scott et al., 1995). Essa considerável variação na resposta à suplementação tem sido atribuída aos diferentes estados fisiológicos das vacas, ao tipo de volumoso da dieta basal, à quantidade total de energia consumida pelo animal sob suplementação e à quantidade e composição da fonte lipídica utilizada (Wu et al., 1991; Gagliostro \& Chilliard, 1992).

Quanto às fontes, lipídios ruminalmente inertes (protegidos), como sais de cálcio de ácidos graxos, têm sido uma boa alternativa e seu uso pode se tornar rotineiro em muitos sistemas de produção (NRC, 2001). Porém, em razão do aumento no custo dos insumos e da redução das margens de lucro da atividade leiteira, têm sido estudadas outras fontes lipídicas interessantes do ponto de vista econômico que amenizam o balanço energético negativo da fase inicial da lactação e que possuem pouco impacto sobre o metabolismo do rúmen.

Este trabalho foi realizado com o propósito de estudar o efeito da inclusão de três diferentes fontes lipídicas (gordura protegida - sais de cálcio de ácidos graxos de óleo de palma e farelo de arroz integral associado a óleo de arroz ou sebo bovino) em dietas com teores semelhantes de proteína, fibra e lipídios sobre o consumo voluntário de matéria seca, a produção de leite e a eficiência alimentar de vacas Jersey na fase inicial de lactação.

\section{Material e Métodos}

O experimento foi realizado no Sistema de Pecuária de Leite (SISPEL) na Estação Experimental Terras Baixas (ETB) do Centro de Pesquisa Agropecuária de Clima Temperado da Empresa Brasileira de Pesquisa Agropecuária (EMBRAPA), localizado no município de Capão do Leão, RS. Foram utilizadas oito vacas Jersey de alto potencial genético, multíparas (3 e 4 a ordens de parição), na fase inicial da lactação (próximo ao pico de lactação), com peso vivo médio de $420 \mathrm{~kg}$ e produção média de $20 \mathrm{~kg}$ de leite por dia. Os animais foram distribuídos ao acaso em dois quadra- dos latinos 4 x 4 , sendo mantidos em dois módulos de um galpão coberto com telhas de zinco. Cada módulo foi dividido em quatro baias equipadas com comedouro, bebedouro com água potável e cama elevada do chão com piso de areia.

Foram estudados os efeitos de quatro tratamentos: $\mathrm{CON}=$ dieta controle, sem a inclusão de lipídios; $\mathrm{GPC}=$ dieta com gordura protegida comercial (sais cálcicos de ácidos graxos de óleo de palma); FAIO = dieta com farelo de arroz integral mais óleo de arroz; e FAIS = dieta com farelo de arroz integral e sebo bovino. Ressalta-se que, na ocasião da execução deste trabalho, ainda não havia a proibição do uso do sebo na alimentação de ruminantes no Brasil (Instrução Normativa $\mathrm{n}^{\circ} 8$ de 25 de março de 2004 do Ministério da Agricultura, Pecuária e do Abastecimento) e seu emprego teve como propósito reduzir o grau de insaturação do óleo do farelo de arroz integral.

Utilizou-se alimentação volumosa constituída de alfafa (Medicago sativa) na forma de feno picado (2 a $4 \mathrm{~cm}$ ) e planta de milho (Zea maiz) na forma de silagem, em quantidades aproximadamente equivalentes, na matéria seca. Os concentrados foram elaborados no local, utilizando-se triturador de grãos, balanças e misturador vertical. O sebo utilizado no tratamento FAIS foi aquecido até tornar-se líquido e, posteriormente, foi aspergido sobre a mistura dos demais ingredientes do concentrado. Após a mistura, os concentrados foram ensacados, identificados e armazenados para posterior fornecimento aos animais. A participação quantitativa dos ingredientes e a composição bromatológica das dietas são apresentadas nas Tabelas 1 e 2, respectivamente.

Os concentrados foram fornecidos separadamente do volumoso três vezes ao dia, após cada ordenha e por volta do meio-dia. A proporção volumoso:concentrado foi regulada para que o consumo dos animais fosse mantido na relação aproximada de 55:45. Durante os períodos de coleta de dados, o feno e a silagem foram amostrados diariamente formando-se uma amostra composta por período experimental. As amostras de silagem foram acondicionadas em sacos plásticos identificados e armazenadas em congelador $\left(-18^{\circ} \mathrm{C}\right)$. As sobras de volumosos foram pesadas diariamente antes da alimentação da manhã para o ajuste da quantidade a ser fornecida.

Os períodos experimentais foram de 28 dias, com 14 dias de período preliminar, com coletas de amostras (alimentos oferecidos, sobras e de leite) efetuadas dos 15 aos 28 dias. As vacas foram ordenhadas duas vezes ao dia, às $7 \mathrm{~h} 30$ e $17 \mathrm{~h} 30$, com registro diário das produções, retirando-se amostras proporcionais de cada ordenha para determinação dos teores 
Tabela 1 - Composição das dietas (kg de matéria seca/dia) experimentais, calculadas de acordo com o NRC $(1989)^{1}$

Table 1 - Ingredient composition ( $k g$ of DM/ day) of the experimental diets calculated according to NRC (1989)

\begin{tabular}{llllc}
\hline Ingrediente $\begin{array}{l}\text { Tratamento } \\
\text { Ingredient }\end{array}$ & \multicolumn{4}{c}{ Treatment } \\
\cline { 2 - 5 } & CON & GPC & FAIO & FAIS \\
& $R P F$ & RBRO & $R B T$ \\
\end{tabular}

Volumosos

Forages

\begin{tabular}{lcccc}
$\begin{array}{l}\text { Alfafa, feno } \\
\text { Alfalfa hay }\end{array}$ & 4,18 & 4,18 & 4,18 & 4,18 \\
$\begin{array}{l}\text { Milho, silagem } \\
\text { Corn silage }\end{array}$ & 4,18 & 4,18 & 4,18 & 4,18 \\
\hline $\begin{array}{l}\text { Concentrado } \\
\text { Concentrate }\end{array}$ & & & & \\
$\begin{array}{l}\text { Milho, moído } \\
\text { Ground corn }\end{array}$ & 4,25 & 3,45 & 2,25 & 2,83 \\
$\begin{array}{l}\text { Soja, farelo } \\
\text { Soybean meal }\end{array}$ & 2,39 & 2,50 & 2,35 & 2,33 \\
$\begin{array}{l}\text { Trigo, farelo } \\
\text { Wheat bran }\end{array}$ & - & 0,39 & - & - \\
$\begin{array}{l}\text { Gordura protegida } \\
\text { Rumen-protected fat }\end{array}$ & - & 0,58 & - & - \\
$\begin{array}{l}\text { Arroz, farelo integral } \\
\text { Rice bran }\end{array}$ & - & - & 2,55 & 1,40 \\
$\begin{array}{l}\text { Óleo de arroz } \\
\text { Rice oil }\end{array}$ & - & - & 0,11 & - \\
$\begin{array}{l}\text { Sebo } \\
\text { Tallow } \\
\text { Calcário } \\
\text { Limestone }\end{array}$ & - & - & - & 0,27 \\
$\begin{array}{l}\text { Fosfato bicálcico } \\
\text { Dicalcium phosphate }\end{array}$ & 0,18 & 0,18 & 0,08 & 0,10 \\
$\begin{array}{l}\text { Mistura min. e vit. } \\
\text { Min.-vit. premix }\end{array}$ & 0,07 & 0,07 & 0,07 & 0,07 \\
$\begin{array}{l}\text { Total } \\
\text { Con - detacontro, }\end{array}$ & 15,32 & 15,60 & 15,97 & 15,53 \\
\hline
\end{tabular}

CON - dieta controle, sem inclusão de lipídios; GPC - dieta com gordura protegida comercial (sais de cálcio de ácidos graxos de óleo de palma); FAIO - dieta com farelo de arroz integral e óleo de arroz; FAIS - dieta com farelo de arroz integral e sebo bovino.

$C O N$ - control diet without fat supplementation, RPF - basal diet with rumen-protected fat, $R B R O$ - basal diet with rice bran and rice oil and RBT - basal diet with rice bran and tallow. ${ }^{1}$ Vacas: peso vivo médio de $420 \mathrm{~kg}$; produção média de $20 \mathrm{~kg} / \mathrm{dia}$ com $4,8 \%$ de gordura (Cows: average body weight of $420 \mathrm{~kg}$; milk yield of $20 \mathrm{~kg} /$ day with $4.8 \%$ of fat).

2 Megalac ${ }^{\circledR}$

3 Fosfocálcio ${ }^{\circledR}$

${ }^{4}$ Bovigold $^{\circledR}$.

de gordura. Os animais foram pesados individualmente, durante dois dias consecutivos, após a ordenha da manhã, antes de voltarem para as baias e receberem a alimentação, nos dias 27 e 28 de cada período experimental, empregando-se a média dos dois dias consecutivos.

As amostras de silagem e de sobras de volumoso de cada período de avaliação foram descongeladas à temperatura ambiente, homogeneizadas, subamostradas e présecas em estufa com ventilação a $55^{\circ} \mathrm{C}$ por 72 horas. Essas amostras, assim como as de feno e de concentrados, foram trituradas em moinho do tipo Wiley com peneira de $1 \mathrm{~mm}$,
Tabela 2 - Teores médios de MS, CIN, PB, EE, FDN, CNF e Ca das dietas experimentais

Table 2 - Average contents of DM, ash, CP, EE, NDF, NFC, and Ca of the experimental diets

\begin{tabular}{|c|c|c|c|c|}
\hline \multirow{3}{*}{$\begin{array}{l}\text { Componente } \\
\text { Component }\end{array}$} & \multicolumn{4}{|c|}{ Tratamento $^{1}$} \\
\hline & \multicolumn{4}{|c|}{ Treatment } \\
\hline & $\mathrm{CON}$ & GPC & $\begin{array}{l}\text { FAIO } \\
\text { RBRO }\end{array}$ & $\begin{array}{c}\text { FAIS } \\
R B T\end{array}$ \\
\hline $\mathrm{MS}, \%(D M, \%)$ & 57,25 & 57,14 & 57,57 & 57,62 \\
\hline$\%$ of $D M$ & \multicolumn{4}{|c|}{$\ldots \%$ da MS } \\
\hline CIN (Ash) & 8,02 & 8,20 & 8,83 & 8,80 \\
\hline $\mathrm{PB}(C P)$ & 17,75 & 17,75 & 17,78 & 17,71 \\
\hline $\mathrm{EE}$ & 2,68 & 5,77 & 5,63 & 5,67 \\
\hline FDN (NDF) & 30,68 & 30,79 & 32,12 & 31,67 \\
\hline $\mathrm{CNF}^{*}(\mathrm{NFC})$ & 40,78 & 37,39 & 35,63 & 36,09 \\
\hline $\mathrm{Ca}$ & 1,07 & 1,39 & 1,15 & 1,17 \\
\hline
\end{tabular}

1 Como descrito na Tabela 1 (as described in Table 1).

* $\mathrm{CNF}=100-(\% \mathrm{CIN}+\% \mathrm{~PB}+\% \mathrm{EE}+\% \mathrm{FDN})$, segundo o NRC (2001).

* NFC $=100-(\% a s h+\% C P+\% E E+\% N D F)$ according to the NRC (2001).

para posterior determinação dos teores de $\mathrm{MS}, \mathrm{CIN}, \mathrm{PB}, \mathrm{EE}$ e FDN. Os teores de $\mathrm{MS}$ foram obtidos a $105^{\circ} \mathrm{C}$; os de CIN, via incineração em mufla a $550^{\circ} \mathrm{C}$ durante 4 horas; os de $\mathrm{PB}$, pelo método Kjeldahl ( $\mathrm{N}$ x 6,25); os de EE, com éter de petróleo em aparelho Goldfish, segundo técnicas descritas na AOAC (1996); e os de FDN, pelo método de Van Soest et al.(1991) com $\alpha$-amilase termo-estável (Termamyl 120L do Laboratório Novozymes Latin America Ltda- PR).

Como não houve sobra dos concentrados, o consumo foi obtido pela diferença entre a oferta e o remanescente da mistura do volumoso (silagem de milho e feno de alfafa) após 24 horas de consumo, ou seja, até a manhã do dia seguinte. A determinação do consumo de MO, PB, EE e FDN foi feita a partir do consumo de MS multiplicado pelos teores de cada fração bromatológica determinada nos alimentos oferecidos, subtraído dos valores correspondentes encontrados nas sobras de volumoso.

A produção de leite foi corrigida para 3,5\% de gordura utilizando-se a produção de leite de cada animal no período e o respectivo teor de gordura do leite, segundo a fórmula $(0,432 \times \mathrm{kg}$ leite $)+(0,1623 \times \mathrm{kg}$ leite $\mathrm{x} \%$ gordura $)$, de Evans et al. (1993).

O modelo estatístico incluiu efeitos do quadrado latino, do animal dentro do quadrado latino, do período, do tratamento e da interação quadrado $\times$ tratamento. Os dados das variáveis estudadas foram submetidos à análise de variância e ao teste Tukey a $5 \%$ de probabilidade pelo procedimento GLM do programa estatístico SAS (1999).

\section{Resultados e Discussão}

Os consumos médios diários de MS e de seus principais constituintes, as relações entre CMS, PV e peso metabólico 
e o consumo de FDN em relação ao peso vivo por tratamento são apresentados na Tabela 3.

Os consumos de MS (CMS), MO (CMO), PB (CPB) e as relações entre CMS, PV e peso metabólico não foram influenciados pela suplementação com lipídios $(\mathrm{P}>0,05)$. Diferentemente, os consumos de EE (CEE) e CNF (CCNF) diferiram $(\mathrm{P}<0,01)$ entre os tratamentos. $\mathrm{O} C F D N$ e a sua relação com o PV diferiram significativamente nos níveis de 0,05 e 0,06 pelo teste $F$, respectivamente.

O CEE foi significativamente inferior nos animais alimentados com a dieta controle, mas não diferiu entre os tratamentos com fontes lipídicas (Tabela 3). O CCNF diferiu significativamente entre o tratamento controle e aqueles com lipídios, observando-se o maior valor nas vacas do tratamento controle, não ocorrendo, porém, diferenças entre as fontes lipídicas. A semelhança $(\mathrm{P}>0,05)$ no $\mathrm{CMO}$ e CPB (Tabela 3 ) pode ser atribuída à ausência de diferença nas concentrações destes cosntituintes nas dietas e à semelhança no CMS entre os tratamentos. As diferenças verificadas no CFDN em $\mathrm{kg} /$ dia e em \% do PV no tratamento FAIO, em relação ao GPC, deve-se à maior participação de FDN na dieta com farelo e óleo de arroz.

Para obtenção de resultados produtivos satisfatórios com a suplementação lipídica, é importante que o CMS não seja reduzido. Caso contrário, dependendo do nível de redução, os efeitos da maior densidade energética proporcionada pela fonte lipídica poderão ser anulados, como verificado por Vargas et al. (2002), que estudaram o efeito do uso de grão de soja moído ou do óleo de soja no nível de $4 \%$ de EE na dieta de vacas produzindo $20 \mathrm{~L}$ de leite/dia e constataram que as fontes lipídicas reduziram em $20 \%$ o CMS, sem alterar a produção de leite.

O consumo médio geral de MS observado, de 16,44 kg/ dia (3,9\% do PV), de acordo com Van Soest (1994), demonstra o bom manejo alimentar e a boa qualidade dos alimentos que compuseram as dietas. Ao contrário do observado em dietas à base de alfafa, segundo Smith et al. (1993) e Onetti et al. (2002), em dietas com volumosos constituídos por níveis elevados de silagem de milho, mesmo de boa qualidade, a suplementação com lipídios não promoveu efeitos positivos na produção de leite.

Excetuando-se os possíveis efeitos das fontes lipídicas utilizadas, não era esperada diferença no consumo voluntário de MS entre os tratamentos, pois as dietas experimentais diferiram da dieta controle basicamente na quantidade de lipídios em detrimento à de CNF, cujos níveis, mesmo naquelas suplementadas, estiveram de acordo com as recomendações do NRC (2001). O mesmo ocorreu com os teores médios de FDN. A quantidade de FDN que uma vaca pode consumir depende do volume do rúmen, que, por sua vez, está relacionado ao peso corporal. A capacidade de consumo de FDN por vacas multíparas tem sido estimada entre 1,0 e $1,3 \%$ do PV (NRC, 2001). O valor médio observado neste trabalho $(1,26 \%)$ foi muito mais próximo do limite superior, demonstrando que a concentração e o tipo de fibra das dietas não comprometeram o consumo voluntário de MS pelos animais experimentais.

A adição das fontes lipídicas estudadas poderia, ainda, provocar redução no consumo voluntário pela diminuição na fermentação ruminal e na digestibilidade da fibra

Tabela 3 - Médias dos consumos de MS (CMS) (em kg/dia, em \% PV e em relação ao peso metabólico - CMS/ PM), MO (CMO), PB (CPB), EE (CEE), FDN (CFDN), CNF (CCNF) e FDN como \%PV nos tratamentos

Table 3 - $\quad$ Means of DM intake (DMI) express in kg/day, as percentage of body weight (DMI, \%BW), and per unit of metabolic body size (DMI/MBS), OM intake (OMI), CP intake (CPI), EE intake (EEI), NDF intake (NDFI), NFC intake (CNFI) and FDNI as \%BW

\begin{tabular}{|c|c|c|c|c|c|c|}
\hline \multirow[t]{2}{*}{$\begin{array}{l}\text { Variável } \\
\text { Item }\end{array}$} & \multicolumn{4}{|c|}{$\begin{array}{c}\text { Tratamento } \\
\text { Treatment }\end{array}$} & \multirow[t]{2}{*}{$\mathrm{P}>\mathrm{F}$} & \multirow[t]{2}{*}{$\mathrm{CV}$} \\
\hline & $\mathrm{CON}$ & $\begin{array}{l}\mathrm{GPC} \\
R P F\end{array}$ & $\begin{array}{l}\text { FAIO } \\
R B R O\end{array}$ & $\begin{array}{c}\text { FAIS } \\
R B T\end{array}$ & & \\
\hline CMS, kg/dia $(D M I, k g / d)$ & 16,48 & 16,33 & 16,71 & 16,23 & 0,3383 & 4,71 \\
\hline $\mathrm{CMS}, \% \mathrm{PV}(D M I, \% B W)$ & 3,95 & 3,89 & 3,90 & 3,90 & 0,8441 & 4,86 \\
\hline $\mathrm{CMS} / \mathrm{PM}(D M I / M B S)$ & 0,178 & 0,176 & 0,180 & 0,176 & 0,4125 & 4,77 \\
\hline $\mathrm{CMO}, \mathrm{kg} / \mathrm{dia}(O M I, \mathrm{~kg} / \mathrm{d})$ & 15,23 & 15,06 & 15,30 & 14,80 & 0,2284 & 4,84 \\
\hline $\mathrm{CPB}, \mathrm{kg} / \mathrm{dia}(C P I, \mathrm{~kg} / \mathrm{d})$ & 2,75 & 2,74 & 2,80 & 2,72 & 0,1061 & 3,31 \\
\hline $\mathrm{CEE}, \mathrm{kg} / \mathrm{dia}(E E I, \mathrm{~kg} / \mathrm{d})$ & $0,45 b$ & $0,91 \mathrm{a}$ & $0,91 \mathrm{a}$ & $0,90 \mathrm{a}$ & 0,0001 & 3,12 \\
\hline CFDN, $\mathrm{kg} / \mathrm{dia}(N D F I, \mathrm{~kg} / \mathrm{d})$ & $5,23 \mathrm{ab}$ & $5,16 b$ & $5,50 \mathrm{a}$ & $5,24 \mathrm{ab}$ & 0,0507 & 6,80 \\
\hline $\mathrm{CCNF}, \mathrm{kg} / \mathrm{dia}(N F C I, \mathrm{~kg} / \mathrm{d})$ & $6,78 \mathrm{a}$ & $6,19 b$ & $6,08 \mathrm{~b}$ & $6,00 \mathrm{~b}$ & 0,0001 & 6,36 \\
\hline $\mathrm{CFDN}, \% \mathrm{PV}(N D F I, \% B W)$ & $1,25 \mathrm{ab}$ & $1,23 b$ & $1,31 \mathrm{a}$ & $1,26 a b$ & 0,0620 & 6,89 \\
\hline
\end{tabular}

Médias, na mesma linha, seguidas de letra diferente diferem $(P<0,05)$ pelo teste Tukey

CV $(\%)=$ coeficiente de variação.

1 Como descrito na Tabela 1 (as described in Table 1).

Means, in the same row, followed by different letter, differ $(P<0.05)$ by Tukey test.

CV $(\%)=$ coeficient of variation 
(Palmquist \& Jenkins, 1980; Chalupa et al., 1986), aumentando o tempo de permanência dos alimentos no rúmenretículo (Allen, 2000). No entanto, resultados obtidos por Nörnberg et al. (2004) indicam que as fontes lipídicas usadas neste trabalho, nos níveis empregados, não afetaram $(\mathrm{P}>0,05)$ a digestibilidade da MS, MO, PB, CNF e FDN.

Além desses aspectos, as fontes lipídicas testadas poderiam ainda afetar o consumo pela ação direta sobre os hormônios intestinais, pela oxidação de ácidos graxos no fígado e pela aceitabilidade das fontes empregadas (Allen, 2000), o que também não ocorreu.

Os resultados deste estudo estão de acordo com os relatos de vários autores, que também não observaram efeito no consumo de MS ao suplementarem dietas com diferentes fontes lipídicas (Elliott et al., 1993; Grummer et al., 1993; Smith et al., 1993; Drackley et al., 1994; Pantoja et al., 1994; Jenkins et al., 1998). Entretanto, divergem dos resultados encontrados em outras pesquisas, nas quais a inclusão de lipídios nas dietas causou redução (Garcia-Bojalil et al., 1998; Onetti et al., 2002; Vargas et al., 2002) ou aumentos no consumo (Pantoja et al., 1996).

Constam na Tabela 4 os valores referentes à produção de leite (sem e com correção a $3,5 \%$ de gordura) e à eficiência alimentar, calculada a partir da produção de leite (sem e com correção para 3,5\% de gordura) em relação ao CMS por tratamento.

A produção de leite foi maior $(\mathrm{P}<0,05)$ nos tratamentos com adição de lipídios e não diferiu significativamente entre as fontes lipídicas estudadas $(\mathrm{P}>0,05)$. As diferenças em relação ao tratamento controle corresponderam a aumentos de 9,$06 ; 7,61$ e $5,60 \%$ para GPC, FAIO e FAIS, respectiva- mente. Quando a produção de leite foi expressa na forma corrigida para mesmo teor de gordura (PLC 3,5\%G), em razão das variações nos seus percentuais, a produção no tratamento GPC permaneceu superior $(\mathrm{P}<0,05)$ à do tratamento controle, ao passo que, nos tratamentos FAIO e FAIS, as produções não diferiram entre si nem em relação aos demais tratamentos $(\mathrm{P}>0,05)$. Nesta comparação, a adição de GPC à dieta promoveu aumento de $1,32 \mathrm{~kg} /$ dia na produção de leite, o que corresponde a $6,5 \%$.

A comparação dos efeitos de tratamentos na produção de leite é feita, há muitas décadas, por meio da produção corrigida para mesmo teor de gordura. Este procedimento é plenamente aceito, visto que a gordura é o componente do leite mais influenciado por mudanças na dieta (Sutton, 1989). Além disso, sua elevada concentração energética demanda quantidade equivalente de energia líquida (EL) para sua síntese.

Supondo uma PL constante, o teor de gordura é o principal fator determinante da quantidade de EL direcionada à produção de leite pelo animal. Entretanto, atualmente, a remuneração ao produtor por parte das indústrias de laticínios desestimula a produção de leite com teores elevados de gordura. Ao mesmo tempo, pesquisas têm sido realizadas visando reduzir a concentração de gordura no leite e aumentar o teor de ácidos graxos insaturados, especialmente de ácidos graxos linoléico conjugados, os quais têm mostrado benefícios à saúde humana (Parodi, 1999). Portanto, a redução na concentração de gordura do leite, desde que não seja decorrente de alteração nos padrões fermentativos, a ponto de comprometer a saúde do animal, pode ser vantajosa tanto do ponto de vista metabólico como econô-

Tabela 4 - Média da produção de leite (PL) corrigida ou não para 3,5\% de gordura (PLC 3,5\%G) e da eficiência alimentar, em kg de leite/kg MS consumida (EFAL) e $\mathrm{kg}$ de leite corrigido para 3,5\% de gordura/kg de MS consumida (EFAL $3,5 \% \mathrm{G}$ )

Table 4 - Means of milk yield (MY), 3.5\% fat-corrected milk (FCM), and feed efficiency (FE) as kg of milk yield/kg of dry matter intake (FE) and kg of $3.5 \%$ FCM $/ \mathrm{kg}$ of DM intake (3.5\% FE)

\begin{tabular}{|c|c|c|c|c|c|c|}
\hline \multirow[t]{2}{*}{$\begin{array}{l}\text { Variável } \\
\text { Item }\end{array}$} & \multicolumn{4}{|c|}{$\begin{array}{l}\text { Tratamento } \\
\text { Treatment }\end{array}$} & \multirow[t]{2}{*}{$\mathrm{P}>\mathrm{F}$} & \multirow[t]{2}{*}{$\mathrm{CV}$} \\
\hline & $\mathrm{CON}$ & $\begin{array}{l}\mathrm{GPC} \\
R P F\end{array}$ & $\begin{array}{l}\text { FAIO } \\
R B R O\end{array}$ & $\begin{array}{c}\text { FAIS } \\
R B T\end{array}$ & & \\
\hline $\begin{array}{l}\text { PL }(\mathrm{kg} / \mathrm{dia}) \\
M Y(\mathrm{~kg} / \mathrm{d})\end{array}$ & $15,89 b$ & $17,33 \mathrm{a}$ & $17,10 \mathrm{a}$ & $16,78 \mathrm{a}$ & 0,0004 & 5,53 \\
\hline $\begin{array}{l}\text { PLC } 3,5 \% \mathrm{G}(\mathrm{kg} / \mathrm{dia}) \\
3.5 \% \text { FCM }(\mathrm{kg} / \mathrm{d})\end{array}$ & $20,37 b$ & $21,69 \mathrm{a}$ & $20,85 \mathrm{ab}$ & $21,07 \mathrm{ab}$ & 0,0148 & 5,33 \\
\hline 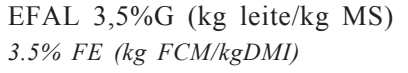 & 1,23 & 1,33 & 1,28 & 1,30 & 0,0861 & 8,34 \\
\hline
\end{tabular}

Médias, na mesma linha, seguidas de letra diferente diferem $(P<0,05)$ pelo teste Tukey

CV $(\%)=$ coeficiente de variação.

${ }^{1}$ Como descrito na Tabela 1 (as described in Table 1).

Means, in the same row, followed by different letter, differ $(P<0.05)$ by Tukey test

CV $(\%)=$ coeficient of variation. 
mico, principalmente no caso de vacas Jersey, cujo teor de gordura do leite é elevado, bem acima dos patamares máximos de bonificação atualmente adotados pelas indústrias brasileiras de laticínios. Assim, respeitados os aspectos nutricionais e visando os benefícios ao produtor, a comparação dos efeitos de fontes lipídicas por meio da produção sem correção para gordura parece adequada, desde que a remuneração ao produtor seja feita com base no volume de leite produzido.

A literatura tem apontado que a adição de lipídios à dieta de vacas lactantes promove efeito quadrático sobre a PL (Jenkins, 1994). Portanto, as quantidades de lipídios na dieta basal e de lipídios suplementar devem sempre ser consideradas. Kronfeld (1976), citado por Hinders (2000), indicou que a PL alcança sua máxima eficiência quando os ácidos graxos constituem 16\% da EM. Segundo Jenkins (1997), para vacas da raça Holandesa, isto equivale a 600$700 \mathrm{~g}$ de lipídios suplementares por dia, entretanto, a resposta máxima observada com adição de lipídios na dieta raramente excede $3,5 \mathrm{~kg}$. Além disso, $700 \mathrm{~g}$ de lipídios seriam necessários para suportar esta produção $(80 \%$ de digestibilidade da fonte lipídica e eficiência de $75 \%$ de utilização pela glândula mamária).

Para vacas da raça Holandesa com PV médio de $600 \mathrm{~kg}$ e consumindo $23 \mathrm{~kg}$ de MS/dia (3,8\% do PV), $700 \mathrm{~g}$ de lipídio suplementar equivalem a aproximadamente $3 \%$ da MS da dieta (Jenkins, 1997). Entretanto, nos animais da raça Jersey utilizados neste trabalho (consumo médio de MS de $16 \mathrm{~kg}$ / dia), a suplementação com $700 \mathrm{~g}$ de lipídios corresponderia à adição de 4,4\% de EE, o que seria elevado em se tratando de fontes lipídicas não-protegidas. Porém, o nível adicional de 3\% de lipídios em relação ao consumo de MS corresponde à quantidade suplementada $(0,46 \mathrm{~kg})$, a qual, de acordo com a relação anterior, suportaria uma produção extra de $2,3 \mathrm{~kg}$ de leite/dia. No entanto, a diferença verificada para suplementação com óleo de palma protegido foi de $1,32 \mathrm{~kg}$ de leite com 3,5\% de gordura/dia. Nas dietas com as outras fontes, as respostas foram menores, de 0,48 e $0,70 \mathrm{~kg}$ de leite para FAIO e FAIS, o que seria atendido com 96 e $140 \mathrm{~g}$ de lipídio suplementar, respectivamente. Portanto, as respostas observadas foram aquém da eficiência estimada na literatura, indicando a necessidade de continuidade de estudos nesse sentido.

Outro fator mencionado na literatura como limitante na identificação de efeitos positivos de fontes lipídicas na PL é o nível médio de produção das vacas. Palmquist \& Jenkins (1980), em uma revisão, constataram efeitos benéficos em vacas produzindo acima de $16 \mathrm{~kg} / \mathrm{dia}(5.000 \mathrm{~kg} / \mathrm{lactação})$. Palmquist (1991) sugeriu que a quantidade total de lipídios na dieta seja igual à de gordura produzida no leite. Todavia, Hinders (2000) recomenda suplementação com lipídios para vacas Jersey somente em caso de produções acima de 25 $27 \mathrm{~kg} / \mathrm{dia}$. Os resultados deste trabalho divergem das recomendações desse autor, com respostas mais condizentes às observações de Palmquist \& Jenkins (1980), tendo em vista que a média de produção no tratamento controle foi de $15,89 \mathrm{~kg} / \mathrm{dia}$ e de $20,37 \mathrm{~kg} / \mathrm{dia}$ quando corrigida para $3,5 \%$ de gordura.

Resultados de pesquisas demonstraram que o sebo tem passagem relativamente inerte pelo rúmen-retículo, mesmo quando usado junto a quantidades consideráveis de óleo proveniente de sementes oleaginosas (Elliott et al., 1993; Wu et al., 1993; Drakley et al., 1994). Considerando essa característa, a disponibilidade regional e o preço, o sebo foi associado ao FAI na expectativa de que a resposta produtiva poderia ser melhorada. Foi constatado, porém, que, em termos produtivos, embora superior ao tratamento controle, a adição de sebo não mostrou vantagem em relação ao tratamento com farelo e óleo de arroz. Entretanto, os resultados comprovaram que o sebo pode ser usado para aumentar a quantidade total de lipídios da dieta com FAI e/ou para corrigir a variação normalmente presente no nível de EE dos farelos disponíveis. Além disso, por meio de observações práticas, a adição de $50 \%$ de sebo $(1,5 \%$ na MS da dieta) também diminuiu a pulverulência do FAI e melhorou o seu manuseio.

O aumento na PL proporcionado pela suplementação com as fontes lipídicas neste trabalho pode ser explicado pelo acréscimo no aporte metabólico de ácidos graxos préformados da dieta. Neste caso, a suplementação possibilitou maior captação de ácidos graxos de cadeia longa pela glândula mamária, disponibilizando maior quantidade de glicose para as células produtoras de leite, pois, para a síntese de ácidos graxos de cadeias curta e média, a glândula mamária necessita de glicose como fornecedora de agentes redutores NADPH, via ciclo das pentoses (Palmquist \& Jenkins, 1980; Gagliostro \& Chilliard, 1992). A maior diferença observada no tratamento com óleo de palma, evidenciada na avaliação da produção de leite corrigida para 3,5\% de gordura, deve-se, provavelmente, à sua maior digestibilidade $(91 \%)$ em relação às demais fontes testadas $(82 \%)$, conforme observado por Nörnberg et al. (2004).

Os resultados observados com FAI associado a óleo ou sebo foram semelhantes aos reportados por Wilks et al. (1991), que compararam os efeitos do uso de sementes de algodão e FAI em dietas com diferentes níveis de amido, em comparação a uma dieta controle, sem suplementação lipídica, em vacas da raça Holandesa. A dieta controle foi 
constituída de $30 \%$ de silagem de milho, $10 \%$ de feno de alfafa e $60 \%$ de concentrado, totalizando 3,8\% de EE. O nível de farelo de arroz foi de $15 \%$ (25\% no concentrado) totalizando 5,9\% de EE na dieta. O consumo de MS não diferiu estatisticamente entre os tratamentos $(21,0 ; 22,5 \mathrm{e}$ $22,0 \mathrm{~kg}$ /dia para a dieta controle, com semente de algodão e FAI, respectivamente), mas a PL corrigida para 3,5\% de gordura tendeu a ser maior $(\mathrm{P}<0,10)$ nos animais alimentados com as dietas contendo farelo de arroz integral $(28,6 \mathrm{~kg} /$ dia) e semente de algodão $(28,0 \mathrm{~kg} /$ dia $)$ em relação ao tratamento controle $(27,8 \mathrm{~kg} / \mathrm{dia})$.

Neste experimento, a eficiência alimentar calculada por meio da PL sem correção para gordura mostrou diferença significativa $(\mathrm{P}<0,05)$, com vantagem para a suplementação com óleo de palma protegido. Contudo, os tratamentos FAIO e FAIS não diferiram entre si e GPC $(\mathrm{P}>0,05)$, apresentando valores numéricos superiores ao grupo controle (Tabela 4). Quando a eficiência alimentar foi medida pela produção corrigida para $3,5 \%$ de gordura, a análise de variância não acusou diferença significativa entre os tratamentos ( $\mathrm{P}>0,05)$, com valores de 1,23; 1,33; 1,28 e 1,30 para os tratamentos CON, GPC, FAIO e FAIS, respectivamente. Esses valores com distribuição numérica, que guarda relação semelhante à produção de leite corrigida, mostraram melhora $(\mathrm{P}<0,0861)$ com a suplementação lipídica, que foi superior em 8,$13 ; 4,06$ e 5,69\% para GPC, FAIO e FAIS, respectivamente. Os resultados divergem dos obtidos por Wu et al. (1993) e Simas et al. (1997), que não observaram diferença ao compararem a suplementação com fontes lipídicas a um tratamento sem suplementação. Todavia, são semelhantes aos reportados por Pantoja et al. (1996), que verificaram maior eficiência alimentar em vacas da raça Holandesa consumindo fontes lipídicas com diferentes graus de saturação $(1,61)$ em relação àquelas sem suplementação $(1,52)$. Os autores atribuíram esse efeito à maior eficiência de utilização da energia da fonte lipídica ou maior concentração de EL para lactação destas dietas.

\section{Conclusões}

Gordura protegida na forma de sais de cálcio de ácidos graxos de óleo de palma e farelo de arroz integral, associados a óleo de arroz ou sebo bovino, totalizando 6\% de EE na MS de dietas com volumosos à base de feno de alfafa e silagem de milho e concentrados à base de milho e farelo de soja (32\% de FDN e 36,5\% de CNF na dieta total), não afetam o consumo voluntário de MS e promovem maior PL. A gordura protegida proporciona também maior PL corrigida para gordura e melhora a eficiência alimentar. Entretanto, independentemente da fonte lipídica estudada, as respostas produtivas foram aquém da eficiência relatada na literatura, indicando a necessidade de mais estudos sobre o assunto.

\section{Agradecimento}

Ao Centro de Pesquisa Agropecuária de Clima Temperado - EMBRAPA, pela infra-estrutura e pelos recursos disponibilizados para realização deste trabalho, e à Mercatho Representações Ltda., pelo fornecimento da gordura protegida (Megalac - Church and Dwight Company).

\section{Literatura Citada}

ALLEN, M.S. Effects of diet on short-term regulation of feed intake by lactating dairy cattle. Journal of Dairy Science, v. 83, n.7, p.1598-1624, 2000.

ASSOCIATION OF OFFICIAL ANALYTICAL CHEMISTS - AOAC. Official methods of analysis. 16.ed. Arlington: 1996. 1137p.

CHALUPA, W.; VECCHIARELLI, B.; ELSER, A.E. et al. Ruminal fermentation in vivo as influenced by long chain fatty acids. Journal of Dairy Science, v.69, n.5, p.1293-1301, 1986

CHILLIARD, Y. Dietary fat and adipose tissue metabolism in ruminants, pigs, and rodents: a review. Symposium advances in ruminant lipid metabolism. Journal of Dairy Science, v.76, n.12, p.3897-3931, 1993.

DOREAU, M.; CHILLIARD, Y. Digestion and metabolism of dietary fat in farm animals. British Journal of Nutrition, v.78, p.1535, 1997 (suppl. 1).

DOREAU, M.; CHILLIARD, Y.; BAUCHART, D. et al. Influence of different fat supplements on digestibility and ruminal digestion in cows. Annales de Zootechnie, v.40, n.1, p.19-30, 1991.

DRACKLEY, J.K.; GRUM, D.E.; McCOY, G.C. et al. Comparison of three methods for incorporation of liquid fat into diets for lactating dairy cows. Journal of Dairy Science, v.77, n.8, p.1386-1484, 1994.

ELLIOTT, J.P.; DRACKEY, J.K.; SCHAUFF, D.J. et al. Diets containing high oil corn and tallow for dairy cows during early lactation. Journal of Dairy Science, v.76, n.3, p.775-789, 1993.

EVANS, E.H.; YORSTON, S.A.; BINNENDYK, D.V. Numerous factors affect milk protein percentage. Feedstuffs, n.15, p.14-21, 1993.

GAGLiOstro, G.A.; CHILliard, Y. Utilizacion de lípidos protegidos en la nutricion de la vaca lechera. I. Efectos sobre la producción y la composición de la leche y sobre la ingestón de materia seca y energía. Revista Argentina de Produção Animal, v.12, n.1, p.1-15, 1992.

GARCIA-BOJALIL, C.M.; STAPLES, C.R.; RISCO, C.A. et al. Protein degradability and calcium salts of long-chain fatty acids in the diets of lactating dairy cows. Production responses. Journal of Dairy Science, v.81, n.5, p.1374-1384, 1998.

GARNSWORTHY, P.C. Fats in dairy cow diets. In: GARNSWORTHY, P.C.; COLE, D.J.A. (Eds.) Recent advances in animal nutrition. Nottingham: University of Nottingham, 1997. p. 87-103.

GRUMMER, R.R; LUCK, M.L; BARMORE, J.A. Rumen fermentation and performance of cows fed roasted soybeans and tallow. Journal of Dairy Science, v.76, n.9, p.26742681, 1993.

HINDERS, R. Optimum dietary fat levels for high-producing cows explored. Feedstuffs, n.13, p.10-27, 2000.

JENKINS, T.C. Regulation of lipid metabolism in the rumen. The Journal of Nutrition, v.124, n.9, p.1372-1376, 1994. 
JENKINS, T.C. Success of fat in dairy rations depends on the amount. Feedstuffs, n.13, p.11-12, 1997.

JENKINS, T.C.; BERTRAND, J.A.; BRIDGES JR., W.C. Interactions of tallow and hay particle size on yield and composition of milk from lactating Holstein cows. Journal of Dairy Science, v. 81, n.5, p.1396-1402, 1998.

NATIONAL RESEARCH COUNCIL - NRC. Nutrient requirements of dairy cattle. 6.ed. Washington, D.C.: National Adacemy of Sciences, 1989. 157p.

NATIONAL RESEARCH COUNCIL - NRC. Nutrient requirements of dairy cattle. 7.ed. Washington, DC.: National Adacemy of Sciences, 2001. 381p.

NÖRNBERG, J.L.; STUMPF JR., W.; LÓPEZ, J. et al. Valor do farelo de arroz integral como fonte de gordura na dieta de vacas Jersey na fase inicial da lactação: digestibilidade aparente de nutrientes. Revista Brasileira de Zootecnia, v.33, n.6, p.2412-2421, 2004.

ONETTI, S.G.; SCHAVER, R.D.; McGUIRRE, M.A. et al. Effects of supplemental tallow on performance of dairy cows fed diets with different corn silage:alfalfa silage rations. Journal of Dairy Science, v.85, n.3, p.632-641, 2002.

PALMQUIST, D.L. Influence of source and amount of dietary fat on digestibility in lactating cows. Journal of Dairy Science, v.74, n.4, p.1354-1360, 1991.

PALMQUIST, D.L.; JENKINS, T.C. Fat in lactation ration: Review. Journal of Dairy Science, v.63, n.1, p.1-14, 1980.

PANTOJA, J.; FIRKINS, J.L.; EASTRIDGE, M.L. Fatty acid digestibility and lactation performance by dairy cows fed fats varying in degree of saturation. Journal of Dairy Science, v.79, n.3, p.429-437, 1996.

PANTOJA, J.; FIRKINS, J.L.; EASTRIDGE, M.L. et al. Effects of fat saturation and source of fiber on site of nutrient digestion and milk production by lactating dairy cows. Journal of Dairy Science, v.77, n.8, p.2341-2356, 1994.

PARODI, P.W. Conjugated linoleic acid and other anticarcinogenic agents of bovine milk fat. Journal of Dairy Science, v.82, n.6, p.1339-1349, 1999.

STATISTIC ANALYSIS SYSTEM - SAS. User's guide. Version 6.12 4.ed. Cary: 1999. v.1, 890p.

SCOTT, T.A.; SHAVER, R.D.; ZEPEDA, L. et al. Effects of rumeninert fat on lactation, reproduction, and helth of high producing Holstein herds. Journal of Dairy Science, v.78, n.11, p.24352451, 1995.
SIMAS, J.M.; HUBER, J.T.; THEURER, C.B. et al. Influence of fat source and sorghum grain treatment on performance and digestibilities of high yielding dairy cows. Journal of Dairy Science, v.80, n.11, p.2907-2912, 1997

SMITH, W.A.; HARRIS, JR., B.; Van HORN, H.H. et al. Effects of forage type on production of dairy cows supplemented with whole cottonseed, tallow, and yeast. Journal of Dairy Science, v.76, n.1, p.205-215, 1993.

SUTTON, J.D. Altering milk composition by feeding. Journal of Dairy Science, v.72, n.10, p.2801-2814, 1989.

Van SOEST, P.J. Nutritional ecology of the ruminant. 2.ed. Cornell: Univesrsity Press, 1994. 476p.

Van SOEST, P.J.; ROBERTSON, J.B.; LEWIS, B.A. Symposium: carbohydrate methodology, metabolism, and nutritional implictions in dairy cattle. Methods for dietary fiber, neutral detergent fiber, and nonstarch polysaccharides in relation to animal nutrition. Journal of Dairy Science, v.74, n.10, p.3583-3597, 1991.

VARGAS, L.N.; LANA, R.P.; JHAM, G.N. et al. Adição de lipídeos na ração de vacas leiteiras: parâmetros fermentativos ruminais, produção e composição do leite. Revista Brasileira de Zootecnia, v.31, p.522-529, 2002 (supl. 1).

WILKS, D.L.; COPPOCK, C.E.; BROOKS, K.N. Effects of differences in starch content of diets with whole cottonsed or rice bran on milk casein. Journal of Dairy Science, v.74, n.4, p.1314-1320, 1991

WU, Z.; HUBER, J.T.; SLEIMAN, F.T. et al. Effect of three supplement fat sources on lactation and digestion in dairy cows. Journal of Dairy Science, v.76, n.11, p.3562-3570, 1993.

WU, Z.; OHAJURUKA, O.A.; PALMQUIST, D.L. Ruminal synthesis, biohydrogenation, and digestibility of fatty acids by dairy cows. Journal of Dairy Science, v.74, n.9, p.3025-3034, 1991 